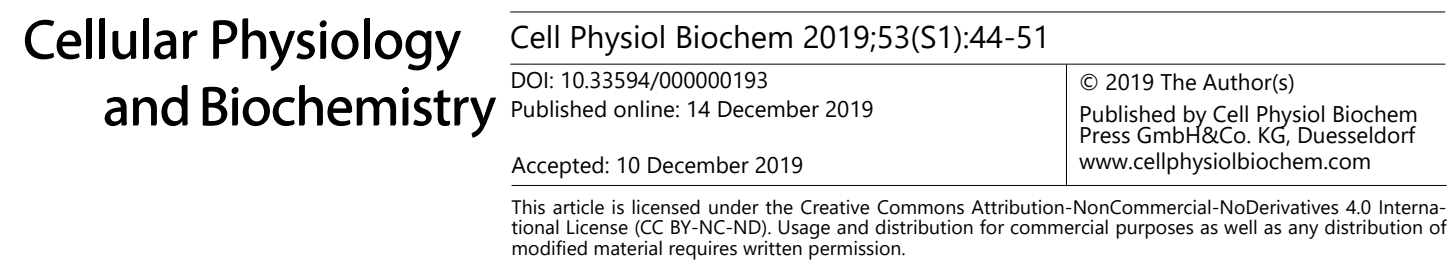

Review

\title{
Intracellular Ion Channels in Pancreas Cancer
}

\author{
Sameer H. Patel Michael J. Edwards Syed A. Ahmad \\ Department of Surgery, University of Cincinnati College of Medicine, Cincinnati, OH, USA
}

\author{
Key Words \\ Ion channels $•$ Pancreas cancer $•$ Oncogenesis
}

\begin{abstract}
The current basis of systemic treatment for pancreas cancer involves cytotoxic chemotherapy. Despite newer regimens, overall survival remains poor and this dilemma is further compounded by a lack of effective, novel therapeutic targets. Another challenge in treating pancreas cancer is the complex tumor microenvironment, which contains regulatory $T$ cells, myeloid derived suppressor cells, and tumor associated macrophages that forms a barrier to standard therapies. Intracellular ion channels are ubiquitously expressed in all cells and their role in carcinogenesis is increasingly becoming elucidated. They play an integral role in each of the six "Hallmarks of Cancer" and are potential novel prognostic biomarkers and therapeutic targets for pancreas cancer. Although examined in various hematologic and gastrointestinal malignancies, there are limited data examining the prognostic role of specific ion channels in pancreas ductal adenocarcinoma. This review focuses on chloride (CLCA-1, CLIC1, CLIC3), calcium (TRPM7, TRPM8), and potassium (Kir3.1, KCa3.1, Kv11.1, Kv1.3) channels in pancreas cancer.
\end{abstract}

\section{Introduction}

Despite the development of more effective systemic therapies and advances in surgical technology, pancreas cancer remains one of the deadliest gastrointestinal malignancies. With an increasing incidence, it is expected to affect 56, 770 patients in the US in 2019 and has moved to the third leading cause of cancer related death [1]. Five year overall survival (OS) remains a dismal 9.3\% for all stages and even for those patients who present with localized disease, it is only $37.4 \%$ [2]. This poor prognosis is attributed to lack of effective screening, late presentation, and high recurrence rates [3]. Of the numerous pancreas cancer histologies, pancreas ductal adenocarcinoma (PDAC) is the most common and will be the focus of this review.

Surgery is the only curative treatment for pancreas cancer, however, systemic therapy is still recommended as the majority of patients have micrometastatic disease at the time of presentation [4]. The CLUSTER study examining circulating tumor cells (CTC) in patients 


\section{Cellular Physiology Cell Physiol Biochem 2019;53(S1):44-51 \\ \begin{tabular}{ll|l} 
and Biochemistry & $\begin{array}{l}\text { DOl: 10.33594/000000193 } \\
\text { Published online: 14 December 2019 }\end{array}$ & $\begin{array}{l}\text { O } 2019 \text { The Author(s). Published by } \\
\text { Cell Physiol Biochem Press GmbH\&Co. KG }\end{array}$ \\
\cline { 2 - 3 } &
\end{tabular} \\ Patel et al.: Ion Channel in PDAC}

with resectable pancreas cancer found that $>95 \%$ of patients had CTCs in peripheral blood samples [3]. This study is one of many showing that patients with seemingly localized pancreas cancer are at high risk of developing distant metastases. The most effective adjuvant systemic therapy regimen, based on published randomized data, is modified FOLFIRINOX. Compared to gemcitabine, FOLFIRINOX was found to have an astonishing median OS of 54.4 mo compared to 35.0 mo [5]. However, despite this newer cytotoxic chemotherapy regimen for PDAC, survival remains poor. One of challenges in treating pancreas cancer is due to the presence of a dense stromal reaction. This stroma contains a multitude of different cell types that result in drug resistance and pro-growth/metastatic signals. Unfortunately, newer drugs specifically targeting the pancreas stroma have resulted in worse outcomes in phase I clinical trials [6].

One of the most exciting treatment strategies to emerge over the last decade has been immunotherapies. Targeting the immune system to fight cancer has shown dramatic results in malignances, such as melanoma. However, similar benefits have not been seen in pancreas cancer due in part to low levels of microsatellite instability and resistance provided by the stroma [7]. Given the lack of new treatments and reliance on still decades old cytotoxic systemic therapies, new and innovative targets are needed.

\section{Ion Channels in Cancer}

Ion channels are an integral part of normal cellular processes regulating cell membrane potential, signal transduction, and proliferation. They are transmembrane proteins found in both the intra- and extracellular cellular compartments that control flow of ions across the cell membrane [8]. The two general categories of ion channels are voltage gated and ligand gated channels. Voltage gated channels are classified based on the ions that passed through them while ligand gated are described based on the ligand that controls the channel [9]. Although ion channels in the plasma membrane have been extensively studied, intracellular ion channels in mitochondria, endoplasmic reticulum, Golgi apparatus, nucleus, lysosomes, endosomes, and peroxisomes are only slowly coming to light [10]. What roles these intracellular channels specifically have in carcinogenesis is only beginning to be understood [11-14].

Hanahan and Weinberg described in their seminal "The Hallmarks of Cancer", that there are six essential processes whereby cancer cells acquire the ability to grow and propagate. They are 1) self-sufficiency in growth signals, 2) insensitivity to antigrowth signals, 3) evading apoptosis, 4) limitless replicative potential, 5) sustained angiogenesis, and 6) tissue invasion and metastasis [15]. Ion channels are intimately involved in all of these oncogenic processes and Prevarskaya et al. eloquently described what roles specific channels have in each process [16]. They further describe that dysfunction of certain channels that results in a malignant phenotype can be considered an "oncochannelopathy" [8]. Despite the extensive role ion channels play, targeting these ubiquitously expressed proteins can be challenging, particularly since normal cells are at risk of substantial toxicity. Strategies to mitigate this risk involves creating a prodrug that can only be activated by cancer cells and targeting channels that are uniquely overexpressed in tumor cells [16].

In pancreas cancer, given the paucity of novel therapeutic targets, ion channels present an untapped field for further study. 


\section{Cellular Physiology Cell Physiol Biochem 2019;53(S1):44-51

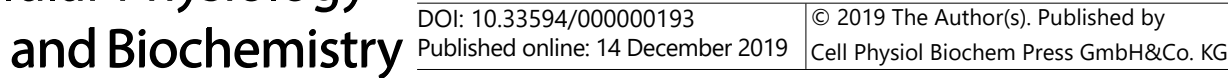 \\ Patel et al.: Ion Channel in PDAC}

\section{Ion Channels in Pancreas Cancer}

As mentioned previously, pancreas cancer presents unique challenges as systemic regimens that are quite effective in other malignancies do not have the same efficacy in pancreas cancer. One of the reasons for this is the dense desmoplastic reaction or stroma associated with pancreas cancer. This stroma can occupy $>80 \%$ of the tumor mass, creating a physical barrier to drug delivery and impairs activation of the immune system $[17,18]$. Once thought to be an immune desert, PDAC is rich in immune cells, however, the majority are immunosuppressive rather than activating. Regulatory $\mathrm{T}$ cells, myeloid derived suppressor cells, and tumor associated macrophages within the stroma work by reducing antigen presentation, inducing $\mathrm{T}$ cell apoptosis, and facilitate transition from a pro- to antiinflammatory state [18]. Numerous clinical trials are underway targeting various components of the stroma. However, of the trials that have completed, the data has not shown the same efficacy that was seen in preclinical studies [19-21]. Ion channels provide an alternative therapeutic target in pancreas cancer that is being increasingly evaluated.

\section{Chloride Channels}

Chloride channels are self-assembling proteins that regulate cell adhesion, control of the cell cycle, osmoregulation, lipid metabolism, mitochondrial function, cell polarity, and apoptosis $[22,23]$. Studies in PDAC have found that not only is there differential expression, but also an association with cancer growth and spread. The Chloride Channel Accessory (CLCA) protein, are self-cleaving proteins that activate Ca-dependent $\mathrm{Cl}^{-}$channels [24]. CLCA specifically interacts with $\beta 4$ integrins and helps facilitate metastatic spread [22]. Of the three functional subtypes, CLCA-1 has been implicated in cell differentiation and carcinogenesis. In colorectal cancer, under expression of this protein has been associated with higher histologic grade, lymph node metastasis, and worse overall survival [25]. Proteomic analysis by Hu et al. examining potential prognostic biomarkers in PDAC showed that CLCA-1 was downregulated by 20 -fold compared to normal tissue [26]. When protein expression was then examined in 140 patients that underwent resection, low CLCA-1 expression was associated with worse disease free survival (11.9 vs 17.5 months, $p=0.042$ ) [24]. On multivariate Cox regression analysis, low CLCA-1 expression remained independently predictive of poorer survival [24]. CLCA-1 also has a potential role in cystic lesions of the pancreas.

PDAC can arise from cystic lesions of the pancreas. The two most commonly implicated are intraductal papillary mucinous neoplasms (IPMNs) and mucinous cystic neoplasms (MCNs). These lesions are considered premalignant and have varying malignant potential, with not all lesions harboring cancer. They are often found incidentally, providing a potential opportunity for early detection and management. However, deciding which lesions require resection, because of underlying high-grade dysplasia or invasive cancer, remains challenging. Cyst fluid sampling and testing for specific protein mutations is an area of active investigation. Studies examining biomarkers in cyst fluid using mass spectroscopy have found that CLCA-1 is secreted into pancreatic cyst fluid [27]. Furthermore, they found that the presence of CLCA-1 carried a $100 \%$ positive predictive value and $87 \%$ negative predictive value for having high-grade dysplasia/invasive cancer [27]. These data suggest that CLCA-1 is not only a potential prognostic biomarker but also a possible therapeutic target in PDAC.

Chloride Intracellular Channels (CLICs) are self-assembling anion channels implicated in regulation of the cell cycle, cell adhesion, migration, tumor invasiveness, and apoptosis $[22,23,28-30]$. These proteins are unique in that they can exist in a soluble form and as an integral membrane protein [23]. CLIC1 was examined in patients with resected PDAC and when compared to adjacent normal tissue, tumor samples had significantly higher protein expression $(67.1 \%$ vs $25.7 \%$, p < 0.001$)$. Furthermore, over expression was associated with higher histologic grade, tumor size, and worse overall survival (HR 5.822, 95\% CI 1.32915.628, p=0.016) [28]. Another CLIC, CLIC3, has also been implicated in PDAC. Studies examining CLIC3 expression in resected PDAC specimens, as well as in precursor lesions called pancreatic intraepithelial neoplasms (PanINs), found overexpression in PDAC 


\section{Cellular Physiology Cell Physiol Biochem 2019;53(S1):44-51 \\ \begin{tabular}{ll|l} 
and Biochemistry $10.33594 / 000000193$ & Published online: 14 December 2019 & $\begin{array}{l}\text { C } 2019 \text { The Author(s). Published by } \\
\text { Cell Physiol Biochem Press GmbH\&Co. KG }\end{array}$
\end{tabular} \\ Patel et al.: Ion Channel in PDAC}

specimens [31]. However, in PanIN lesions, CLIC3 expression increased with greater degrees of dysplasia compared to lesions with well-organized epithelia [31]. When examining survival, high CLIC3 expression was the strongest independent predictor of worse OS (HR 4.81, 95\%CI 1.86-12.3, $\mathrm{p}=0.001$ ] [31]. CLIC3's role in tumor invasiveness is thought to be through a Rab25 dependent mechanism, which is essential for integrin recycling. Taken together, these data show the importance of chloride channels in PDAC invasiveness and progression.

\section{Calcium Channels}

Calcium is one of the most important second messengers in cellular processes. As such, regulation of calcium plays an integral role in carcinogenesis. One of the calcium channel super-families that has a particular role in PDAC is the transient receptor potential (TRP) group. These proteins form either homo- or heterotetramers and are subdivided into seven subfamilies (classical or canonical, melastatin, ankyrin, vanilloid, polycystin, NOMP-C homologues, and mucolipin) [32].

TRPM7, of the melastatin subfamily, has been shown to have a key role in development of the exocrine pancreas in zebrafish [33]. This protein was also examined in patients with resected PDAC and compared to normal controls, TRPM7 was overexpressed in PDAC specimens [33]. The same group then used small interfering RNAs (siRNAs) to TRPM7 in human pancreas cancer cell lines and found this halted cell cycle progression, resulting in reduced number of cells in the $S$ and $\mathrm{G}_{2} / \mathrm{M}$ phases [33]. This suggested that upregulation of TRPM7 drives carcinogenesis by promoting cell cycle progression.

Another calcium channel in the melastatin subfamily that has a role in PDAC is TRPM8. Like TRPM7, TRPM8 is overexpressed in human PDAC specimens compared to normal tissues [34]. Using siRNAs to TRPM8, not only was cell proliferation reduced, but there was evidence of replicative senescence. This was confirmed via SA $\beta$-gal assays, which showed activation in the TRPM8 deficient cells [35]. The exact mechanism of protein upregulation is still underway but the prognostic implications are significant.

The calcium channels TRPM7 and 8 are upregulated in PDAC, and are associated with increased tumor invasiveness, proliferation, and migration [32, 36-38]. Like chloride channels, these calcium channels could be therapeutic targets of anticancer therapies in PDAC.

\section{Potassium Channels}

Potassium channels are one of the most widely studied ion channels in pancreas cancer. They are categorized into four groups: voltage gates (Kv), calcium dependent (KCa), twopore domain group (K2p), and inward rectifying (Kir) [39]. Two channels that are over expressed in pancreas cancer cell lines are Kir3.1 and KCa3.1 [40-42].

Kir3.1 is found within lymphocytes and in resected human PDAC specimens, found to be overexpressed in $80 \%$ of tumor specimens [40]. These findings were confirmed by examining RNA levels using reverse transcriptase polymerase chain reaction. However, when examining clinicopathologic features (metastasis) and protein expression, no significant associations were found [40]. Regardless, this study highlights the importance of potassium channels in PDAC carcinogenesis.

Within the tumor microenvironment, lymphocyte activation is essential in determining how the immune system will respond to cancer. Like Kir3.1, the calcium dependent K-channel, KCa3.1, is found within lymphocytes and can modulate their activity [22]. Examination of protein expression in resected human PDAC specimens, showed overexpression to be associated with advanced cancer stage and shorter survival [22, 43]. Studies examining other calcium activated K-channels in pancreas cancer cell lines have found that in PANC-1 cell lines, which have activating mutations in K-ras, there were significantly less functional KCa channels per cell compared to BxPC-3 and MiaPaCa-2 cells (PANC-1: $\sim 200$ channels/ cell; BxPC-3 600 channels/cell; MiaPaCa-2: 1200 channels/cell) [41]. When these cells were treated with K-channel inhibitors, proliferation of BxPC-3 and MiaPaCa-2 cells 


\section{Cellular Physiology Cell Physiol Biochem 2019;53(S1):44-51 \\ \begin{tabular}{ll|l} 
and Biochemistry & $\begin{array}{l}\text { DOI: } 10.33594 / 000000193 \\
\text { Published online: } 14 \text { December } 2019\end{array}$ & $\begin{array}{l}\text { C } 2019 \text { The Author(s). Published by } \\
\text { Cell Physiol Biochem Press GmbH\&Co. KG }\end{array}$
\end{tabular} \\ Patel et al.: Ion Channel in PDAC}

stopped whereas it was minimally affected in the PANC-1 cell line [41]. These findings show how differential expression of K-channels can influence cell proliferation. When mRNA evaluation of KCa channels in resected human PDAC was performed, $89 \%$ of specimens had upregulation. Compared to the findings from pancreas cancer cell lines (BxPC-3, MiaPaCa-2, and PANC-1), in human specimens, there is overexpression of KCa channels and these could be possible therapeutic targets.

Another voltage gated potassium channel, Kv11.1 (rapid delayed rectifier) was also examined in human PDAC. Immunohistochemical examination of Kv11.1 in 78 PDAC tissue samples found overexpression in tumors compared to the surrounding normal pancreas [44]. When examining clinical features, Kv11.1 overexpression was associated with poor differentiation, higher T-stage, and lymph node positivity [44]. mRNA levels of Kv11.1 was evaluated in human pancreas cancer cell lines and found to be higher compared to nonpancreas cancer cells. In order to examine the mechanism, siRNA silencing of Kv11.1 was undertaken and showed slower cell proliferation in PANC-1 cell lines with higher cell cycles arrest at G1 and greater amounts of apoptosis [44]. Kv11.1 is known to be involved in reorganizing f-actin in PDAC and promoting cell migration [45]. Tumor injection models performed in BALB/c nu/nu mice with si-RNA silenced Kv11.1 found there were significantly lower tumor weights and metastatic nodules in the silenced group [44].

One of the most exciting areas of investigation are the role of specific mitochondrial potassium channels in the development of pancreas cancer. Of these, the voltage dependent potassium channel, Kv1.3, has been studied the most. These topics, as well as the role of mitochondrial Kv1.3 in cancer, will be specifically addressed in other articles within this issue.

\section{Clinical Trials in Pancreas Cancer}

Although there are no specific ion channel inhibitors being examining in clinical trials for PDAC, one promising area of study is use of specific mitochondrial inhibitors. The mitochondria plays an essential role in cell physiology and is home to numerous ion channels located in the inner and outer mitochondrial membranes $[46,47]$. It's role in carcinogenesis is becoming increasingly apparent and is the focus of an ongoing clinical trial.

The novel protein CPI-613 acts by inhibiting pyruvate dehydrogenase and a-ketoglutarate dehydrogenase complexes, thus altering mitochondrial energy metabolism in tumor cells [48, 49]. CPI-613 was evaluated in a phase 1 clinical trial for patients with metastatic pancreas cancer and was found to have an objective response rate of $61 \%$ and complete response rate of $17 \%$ [48]. Currently there is an ongoing phase 3 randomized control trial (NCT03504423) of CPI-613 with modified FOLFIRINOX vs FOLFIRINOX in patients with metastatic pancreas cancer [49]. The data supporting this trial show that targeting the mitochondria in pancreas cancer can result in dramatic responses and further investigations of specific ion channels within the mitochondria may show tremendous promise.

\section{Conclusion}

Intracellular ion channels play an integral role in the process of oncogenesis and only recently are studies beginning to show how they are involved in the "Hallmarks of Cancer". Pancreas cancer remains a challenging malignancy to treat due to resistance mechanisms developed by the cancer and paucity of effective therapeutic targets. However, ion channels present a novel area of investigation and with better understanding of tumor biology, may provide an alternative and effective treatment for pancreas cancer. 


\section{Cellular Physiology Cell Physiol Biochem 2019;53(S1):44-51 \begin{tabular}{ll|l} 
and Biochemistry & $\overline{\text { DOl: 10.33594/000000193 }}$ & $\begin{array}{l}\text { O } 2019 \text { The Author(s). Published by } \\
\text { Published online: } 14 \text { December } 2019\end{array}$ \\
\cline { 2 - 3 } & Cell Physiol Biochem Press GmbH\&Co. KG \\
\hline
\end{tabular}}

\section{Acknowledgements}

\section{Statement of Ethics}

The authors have no ethical conflicts to disclose.

\section{Author Contributions}

S Patel, S Ahmad, and M Edwards were involved in the writing, critical review, and revisions of the manuscript.

\section{Disclosure Statement}

The authors have no conflicts of interest to declare.

\section{References}

1 Siegel RL, Miller KD, Jemal A: Cancer statistics, 2019. CA Cancer J Clin 2019;69:7-34.

2 National Cancer Institute: Surveillance, Epidemiology, and End Results Program (SEER)-Pancreatic Cancer, 2019. URL: https://seer.cancer.gov/.

3 Gemenetzis G, Groot VP, Yu J, Ding D, Teinor JA, Javed AA, Wood LD, Burkhart RA, Cameron JL, Makary MA, Weiss MJ, He J, Wolfgang CL: Circulating Tumor Cells Dynamics in Pancreatic Adenocarcinoma Correlate With Disease Status: Results of the Prospective CLUSTER Study. Ann Surg 2018;268:408-420.

4 National Comprehensive Cancer Network (NCCN): C=Version 3.2019 Pancreatic Adenocarcinoma, 2019.

5 Conroy T, Hammel P, Hebbar M, Ben Abdelghani M, Wei AC, Raoul JL, Chone L, Francois E, Artru P, Biagi JJ, Lecomte T, Assenat E, Faroux R, Ychou M, Volet J, Sauvanet A, Breysacher G, Di Fiore F, Cripps C, Kavan P, et al.: FOLFIRINOX or Gemcitabine as Adjuvant Therapy for Pancreatic Cancer. N Engl J Med 2018;379:23952406.

6 Ramanathan RK, McDonough S, Philip PA, Hingorani SR, Lacy J, Kortmansky JS, Thumar JR, Chiorean EG, Shields AF, Behl D, Mehan PT, Gaur R, Seery TE, Guthrie K, Hochster HS: A phase IB/II randomized study of mFOLFIRINOX (mFFOX) + pegylated recombinant human hyaluronidase (PEGPH20) versus mFFOX alone in patients with good performance status metastatic pancreatic adenocarcinoma (mPC): SWOG S1313 (NCT \#01959139). J Clin Oncol 2018;36:208.

7 Henriksen A, Dyhl-Polk A, Chen I, Nielsen D: Checkpoint inhibitors in pancreatic cancer. Cancer Treat Rev 2019;78:17-30.

8 Prevarskaya N, Skryma R, Shuba Y: Ion Channels in Cancer: Are Cancer Hallmarks Oncochannelopathies? Physiol Rev 2018;98:559-621.

9 Hille B: Ion Channels of Excitable Membranes, ed 3rd. Sunderland, MA, Sinauer, 2001.

$10 \mathrm{Xu} \mathrm{H}$, Martinoia E, Szabo I: Organellar channels and transporters. Cell Calcium 2015;58:1-10.

11 Ko EA, Kim YW, Lee D, Choi J, Kim S, Seo Y, Bang H, Kim JH, Ko JH: Expression of potassium channel genes predicts clinical outcome in lung cancer. Korean J Physiol Pharmacol 2019;23:529-537.

12 Ko JH, Ko EA, Gu W, Lim I, Bang H, Zhou T: Expression profiling of ion channel genes predicts clinical outcome in breast cancer. Mol Cancer 2013;12:106.

13 Wang HY, Li JY, Liu X, Yan XY, Wang W, Wu F, Liang TY, Yang F, Hu HM, Mao HX, Liu YW, Zhang SZ: A three ion channel genes-based signature predicts prognosis of primary glioblastoma patients and reveals a chemotherapy sensitive subtype. Oncotarget 2016;7:74895-74903.

14 Fiske JL, Fomin VP, Brown ML, Duncan RL, Sikes RA: Voltage-sensitive ion channels and cancer. Cancer Metastasis Rev 2006;25:493-500.

15 Hanahan D, Weinberg RA: The hallmarks of cancer. Cell 2000;100:57-70.

16 Prevarskaya N, Skryma R, Shuba Y: Ion channels and the hallmarks of cancer. Trends Mol Med 2010;16:107-121. 


\section{Cellular Physiology Cell Physiol Biochem 2019;53(S1):44-51 \begin{tabular}{l|l|l} 
and Biol: 10.33594/000000193 & (c)19 The Author(s). Published by
\end{tabular} and BiOChemistry Published online: 14 December 2019 Cell Physiol Biochem Press GmbH\&Co. KG \\ Patel et al.: Ion Channel in PDAC}

17 Duluc C, Moatassim-Billah S, Chalabi-Dchar M, Perraud A, Samain R, Breibach F, Gayral M, Cordelier P, Delisle MB, Bousquet-Dubouch MP, Tomasini R, Schmid H, Mathonnet M, Pyronnet S, Martineau Y, Bousquet C: Pharmacological targeting of the protein synthesis mTOR/4E-BP1 pathway in cancer-associated fibroblasts abrogates pancreatic tumour chemoresistance. EMBO Mol Med 2015;7:735-753.

18 Tan E, El-Rayes B: Pancreatic Cancer and Immunotherapy: Resistance Mechanisms and Proposed Solutions. J Gastrointest Cancer 2019;50:1-8.

19 Wolpin BM, Hezel AF, Abrams T, Blaszkowsky LS, Meyerhardt JA, Chan JA, Enzinger PC, Allen B, Clark JW, Ryan DP, Fuchs CS: Oral mTOR inhibitor everolimus in patients with gemcitabine-refractory metastatic pancreatic cancer. J Clin Oncol 2009;27:193-198.

20 Javle MM, Shroff RT, Xiong H, Varadhachary GA, Fogelman D, Reddy SA, Davis D, Zhang Y, Wolff RA, Abbruzzese JL: Inhibition of the mammalian target of rapamycin (mTOR) in advanced pancreatic cancer: results of two phase II studies. BMC Cancer 2010;10:368.

21 Ramanathan RK, McDonough SL, Philip PA, Hingorani SR, Lacy J, Kortmansky JS, Thumar J, Chiorean EG, Shields AF, Behl D, Mehan PT, Gaur R, Seery T, Guthrie KA, Hochster HS: Phase IB/II Randomized Study of FOLFIRINOX Plus Pegylated Recombinant Human Hyaluronidase Versus FOLFIRINOX Alone in Patients With Metastatic Pancreatic Adenocarcinoma: SWOG S1313. J Clon Oncol 2019;37:1062-1069.

22 Arcangeli A, Crociani O, Bencini L: Interaction of tumour cells with their microenvironment: ion channels and cell adhesion molecules. A focus on pancreatic cancer. Philos Trans R Soc Lond B Biol Sci 2014;369:20130101.

23 Anderson KJ, Cormier RT, Scott PM: Role of ion channels in gastrointestinal cancer. World J Gastroenterol 2019;25:5732-5772.

24 Hu D, Ansari D, Zhou Q Sasor A, Hilmersson KS, Bauden M, Jiang Y, Andersson R: Calcium-activated chloride channel regulator 1 as a prognostic biomarker in pancreatic ductal adenocarcinoma. BMC Cancer 2018;18:1096.

25 Yang B, Cao L, Liu J, Xu Y, Milne G, Chan W, Heys SD, McCaig CD, Pu J: Low expression of chloride channel accessory 1 predicts a poor prognosis in colorectal cancer. Cancer 2015;121:1570-1580.

26 Hu D, Ansari D, Pawlowski K, Zhou Q, Sasor A, Welinder C, Kristl T, Bauden M, Rezeli M, Jiang Y, MarkoVarga G, Andersson R: Proteomic analyses identify prognostic biomarkers for pancreatic ductal adenocarcinoma. Oncotarget 2018;9:9789-9807.

27 Jabbar KS, Arike L, Verbeke CS, Sadik R, Hansson GC: Highly Accurate Identification of Cystic Precursor Lesions of Pancreatic Cancer Through Targeted Mass Spectrometry: A Phase IIc Diagnostic Study. J Clin Oncol 2018;36:367-375.

28 Jia N, Dong S, Zhao G, Gao H, Li X, Zhang H: CLIC1 overexpression is associated with poor prognosis in pancreatic ductal adenocarcinomas. J Cancer Res Ther 2016;12:892-896.

29 Peretti M, Angelini M, Savalli N, Florio T, Yuspa SH, Mazzanti M: Chloride channels in cancer: Focus on chloride intracellular channel 1 and 4 (CLIC1 AND CLIC4) proteins in tumor development and as novel therapeutic targets. Biochim Biophys Acta 2015;1848:2523-2531.

30 Lu J, Dong Q, Zhang B, Wang X, Ye B, Zhang F, Song X, Gao G, Mu J, Wang Z, Ma F, Gu J: Chloride intracellular channel 1 (CLIC1) is activated and functions as an oncogene in pancreatic cancer. Med Oncol 2015;32:616.

31 Dozynkiewicz MA, Jamieson NB, Macpherson I, Grindlay J, van den Berghe PV, von Thun A, Morton JP, Gourley C, Timpson P, Nixon C, McKay CJ, Carter R, Strachan D, Anderson K, Sansom OJ, Caswell PT, Norman JC: Rab25 and CLIC3 collaborate to promote integrin recycling from late endosomes/lysosomes and drive cancer progression. Dev Cell 2012;22:131-145.

32 Yee NS, Chan AS, Yee JD, Yee RK: TRPM7 and TRPM8 Ion Channels in Pancreatic Adenocarcinoma: Potential Roles as Cancer Biomarkers and Targets. Scientifica (Cairo) 2012;2012:415158.

33 Yee NS, Zhou W, Liang IC: Transient receptor potential ion channel Trpm7 regulates exocrine pancreatic epithelial proliferation by Mg2+-sensitive Socs3a signaling in development and cancer. Dis Model Mech 2011;4:240-254.

34 Yee NS, Zhou W, Lee M: Transient receptor potential channel TRPM8 is over-expressed and required for cellular proliferation in pancreatic adenocarcinoma. Cancer Lett 2010;297:49-55.

35 Yee NS, Brown RD, Lee MS, Zhou W, Jensen C, Gerke H, Yee RK: TRPM8 ion channel is aberrantly expressed and required for preventing replicative senescence in pancreatic adenocarcinoma: potential role of TRPM8 as a biomarker and target. Cancer Biol Ther 2012;13:592-599. 


\section{Cellular Physiology Cell Physiol Biochem 2019;53(S1):44-51

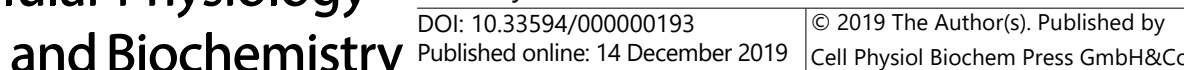 \\ Patel et al.: Ion Channel in PDAC}

36 Lin R, Wang Y, Chen Q Liu Z, Xiao S, Wang B, Shi B: TRPM2 promotes the proliferation and invasion of pancreatic ductal adenocarcinoma. Mol Med Rep 2018;17:7537-7544.

37 Shapovalov G, Ritaine A, Skryma R, Prevarskaya N: Role of TRP ion channels in cancer and tumorigenesis. Semin Immunopathol 2016;38:357-369.

38 Rybarczyk P, Gautier M, Hague F, Dhennin-Duthille I, Chatelain D, Kerr-Conte J, Pattou F, Regimbeau JM, Sevestre H, Ouadid-Ahidouch H: Transient receptor potential melastatin-related 7 channel is overexpressed in human pancreatic ductal adenocarcinomas and regulates human pancreatic cancer cell migration. Int J Cancer 2012;131:E851-861.

39 Felipe A, Vicente R, Villalonga N, Roura-Ferrer M, Martinez-Marmol R, Sole L, Ferreres JC, Condom E: Potassium channels: new targets in cancer therapy. Cancer Detect Prev 2006;30:375-385.

40 Brevet M, Fucks D, Chatelain D, Regimbeau JM, Delcenserie R, Sevestre H, Ouadid-Ahidouch H: Deregulation of 2 potassium channels in pancreas adenocarcinomas: implication of KV1.3 gene promoter methylation. Pancreas 2009;38:649-654.

41 Jager H, Dreker T, Buck A, Giehl K, Gress T, Grissmer S: Blockage of intermediate-conductance Ca2+activated K+ channels inhibit human pancreatic cancer cell growth in vitro. Mol Pharmacol 2004;65:630638.

42 Lang F, Stournaras C: Ion channels in cancer: future perspectives and clinical potential. Philos Trans R Soc Lond B Biol Sci 2014;369:20130108.

43 Jiang S, Zhu L, Yang J, Hu L, Gu J, Xing X, Sun Y, Zhang Z: Integrated expression profiling of potassium channels identifys KCNN4 as a prognostic biomarker of pancreatic cancer. Biochem Biophys Res Commun 2017;494:113-119.

44 Feng J, Yu J, Pan X, Li Z, Chen Z, Zhang W, Wang B, Yang L, Xu H, Zhang G, Xu Z: HERG1 functions as an oncogene in pancreatic cancer and is downregulated by miR-96. Oncotarget 2014;5:5832-5844.

45 Manoli S, Coppola S, Duranti C, Lulli M, Magni L, Kuppalu N, Nielsen N, Schmidt T, Schwab A, Becchetti A, Arcangeli A: The Activity of Kv 11.1 Potassium Channel Modulates F-Actin Organization During Cell Migration of Pancreatic Ductal Adenocarcinoma Cells. Cancers (Basel) 2019;11:pii:E135.

46 Bachmann M, Costa R, Peruzzo R, Prosdocimi E, Checchetto V, Leanza L: Targeting Mitochondrial Ion Channels to Fight Cancer. Int J Mol Sci 2018;19:pii:E2060.

47 Leanza L, Biasutto L, Manago A, Gulbins E, Zoratti M, Szabo I: Intracellular ion channels and cancer. Front Physiol 2013;4:227.

48 Alistar A, Morris BB, Desnoyer R, Klepin HD, Hosseinzadeh K, Clark C, Cameron A, Leyendecker J, D’Agostino R Jr., Topaloglu U, Boteju LW, Boteju AR, Shorr R, Zachar Z, Bingham PM, Ahmed T, Crane S, Shah R, Migliano JJ, Pardee TS, et al.: Safety and tolerability of the first-in-class agent CPI-613 in combination with modified FOLFIRINOX in patients with metastatic pancreatic cancer: a single-centre, open-label, doseescalation, phase 1 trial. Lancet Oncol 2017;18:770-778.

49 Philip PA, Buyse ME, Alistar AT, Rocha Lima CM, Luther S, Pardee TS, Van Cutsem E: A Phase III open-label trial to evaluate efficacy and safety of CPI-613 plus modified FOLFIRINOX ( $\mathrm{mFFX}$ ) versus FOLFIRINOX (FFX) in patients with metastatic adenocarcinoma of the pancreas. Future Oncol 2019;15:3189-3196. 